\title{
THEORY OF EVOLUTION OF THE CENTRAL STAR
}

\author{
Giora Shaviv \\ Department of Physics and Astronomy \\ Te1-Aviv University, Ramat-Aviv, Israel
}

The fundamental feature of the evolution of the central star of planetary nebulae (CPN) is the cooling of the core towards a white dwarf. The basic observations were made by O'Del1 (1963), Seaton (1966), and Harman and Seaton (1964); namely, they discovered a correlation between the linear size of the nebula and the position of the CNP in the HR diagram. Typical values for the velocity of expansion are $10-30 \mathrm{~km} / \mathrm{sec}$ and the largest size observed for a nebula is $\sim 0.6 \mathrm{pc}$ and hence the lifetime is $2-6 \times 10^{4}$ years. From a theoretical point of view the important fact about the Harman-Seaton sequence (HS) is the implication on the evolution of the central star. The star first heats up, $\mathrm{L}$ and Te increase, then $\mathrm{L}$ decreases. But Te keeps increasing until finally Te decreases also (shown schematically in Figure 2). Thus the HS is an evolutionary sequence and not a locus of stars.

The general assumption is that CPN evolve into WD. The observed narrow spread in WD masses implies again that the spread in mass in the HS is small. Definitely, $\Delta \mathrm{m} \ll 0.1 \mathrm{M}_{\odot}$.

The first attempts to explain the evolution of CPN were made by means of homogeneous models. A typical composition assumed is $50 \% \mathrm{C}$ and $50 \% 0$ by mass. Simple theoretical evolutionary tracks are shown in Figures 1 to 3 . A comparison of calculations made by Katz et a1. (1974) and by Kovetz and Shaviv (1976) show that the evolution up to the point of $\left(\mathrm{T}_{\mathrm{e}}\right)_{\max }$ depends critically on the initial conditions and it is merely a transient of the star which follows the mass ejection (see Figure 4). Similar conclusions were arrived at by Deinzer and Sengburch (1970) and Härm and Schwarzschild (1975).

It is obvious that simple homogeneous models are not realistic and fail to mimic the HS sequence. If the total mass is increased the effective temperature increases as well. If, however, one tries to lower the mass so as to fit the effective temperature, the luminosity is too low. The time scale does not pose a problem because of intensive v-emission. 


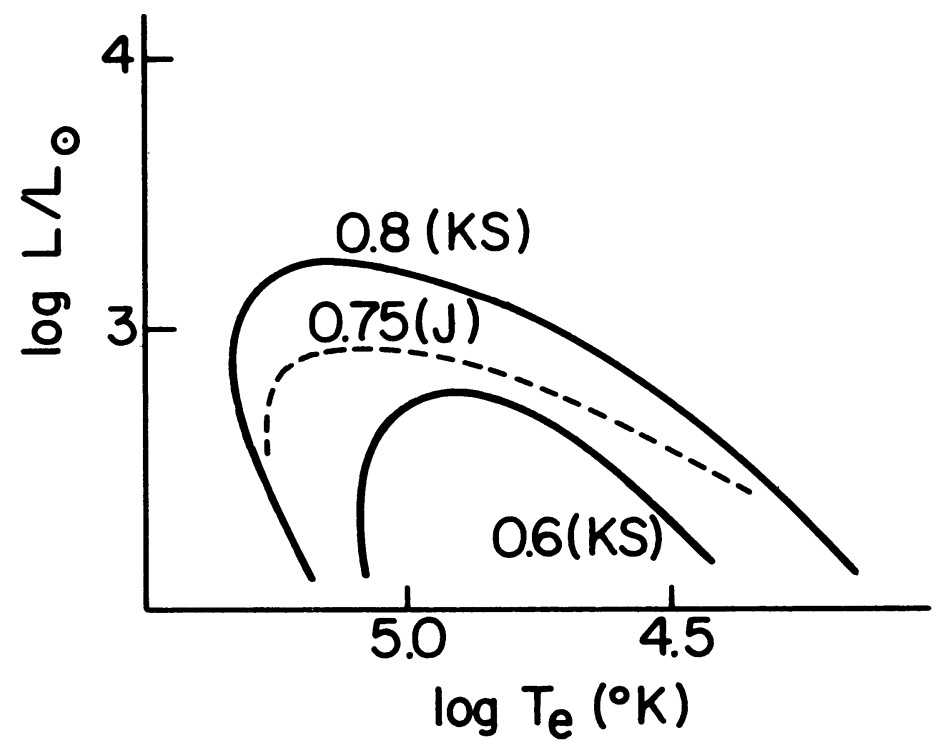

Figure 1. Comparison between the evolution of homogeneous C/O curves with different initial conditions. KS - calculations by Kovetz and Shaviv (1976); J - calculations by Joss et al (1973).

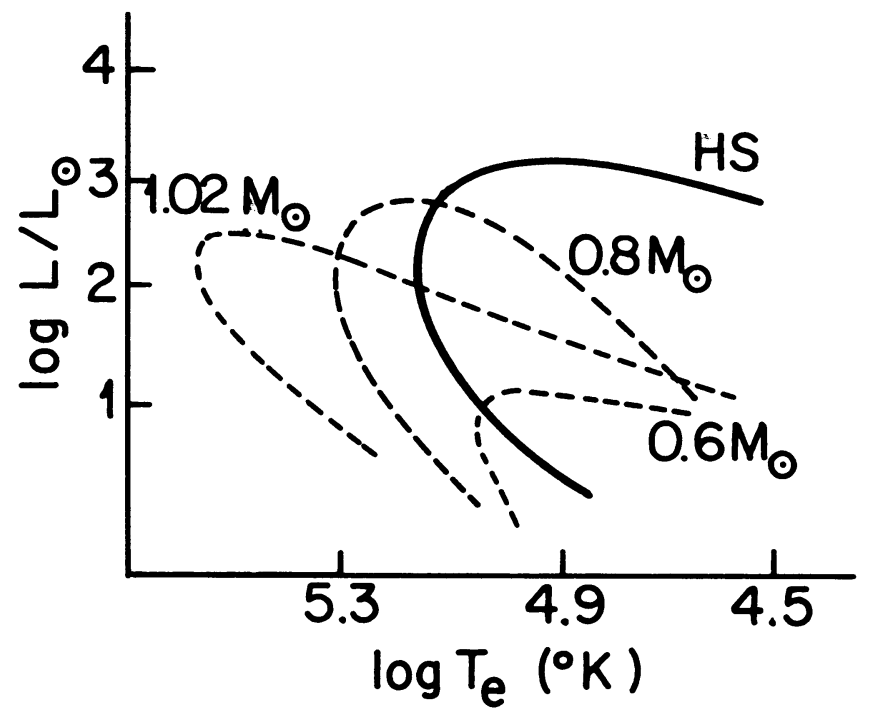

Figure 2. Comparison between the observed HS sequence and the evolution of homogeneous models (dashed curves). The numbers indicate the assumed core mass of $\mathrm{C} / 0$. 
The next attempt was made by Paczyński (1971) who assumed very small amounts of hydrogen and helium. When the amount of hydrogen-rich material is smal1, the hydrogen shell flashes just once and the star makes a small loop in the HR diagram. So long as the amount of hydrogen is smal1, the evolution is at constant bolometric luminosity. The reason for this is that in this range of envelope masses and core masses, the average luminosity depends only on the core mass.

The next attempt by Katz et al. (1974) was to increase the amount of hydrogen. There is however a basic difference between the calculations by Paczyński on the one hand and Katz et al. on the other. The first assumed a sma11 $\mathrm{H}$ envelope and complete thermal equilibrium as initial conditions while the second assumed low $\rho$, low $\mathrm{T}$ and nondegenerate core as initial condition. The flash obtained by Paczyński is very flat and short compared to the assumed lifetime in the HS phase, while the flashes obtained by Katz et al. are very high (in L) and their shape depends critically on the amount of $\mathrm{H}$ mass (see Figure 4).

It is clear that both assumptions are incorrect because the time scale for mass 1 oss $\simeq$ time scale for thermal relocation $\simeq$ lifetime on the HS sequence. Consequently, the star will not be in thermal equilibrium after the end of the mass-loss episode and before going on the HS sequence. Moreover, the exact detail of the upper part of the HS sequence depends critically on the way the mass loss ended. As an example we can see the results by Härm and Schwarzschild (1975). The authors removed the outer shells artificially and obtained at the end a star with very low luminosity (by a factor of 10).

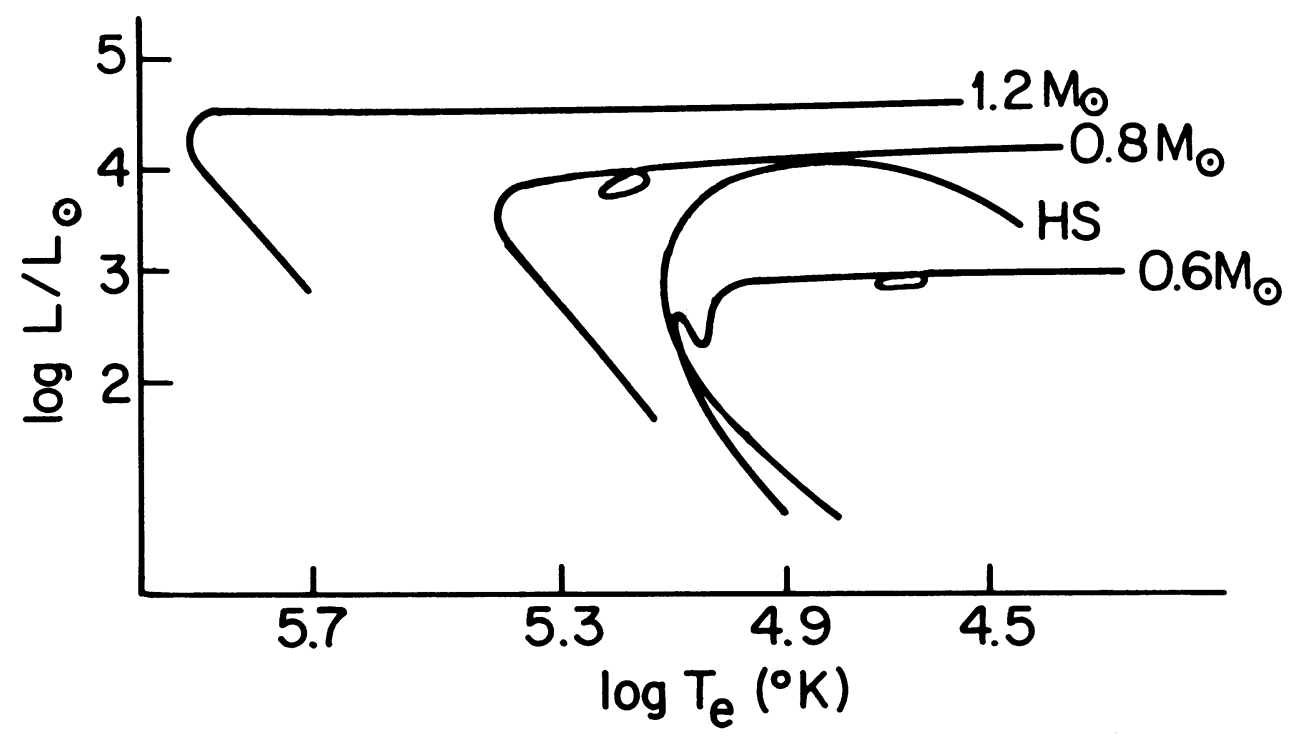

Figure 3. Comparison between the HS and models with small amounts of hydrogen-rich envelopes. 


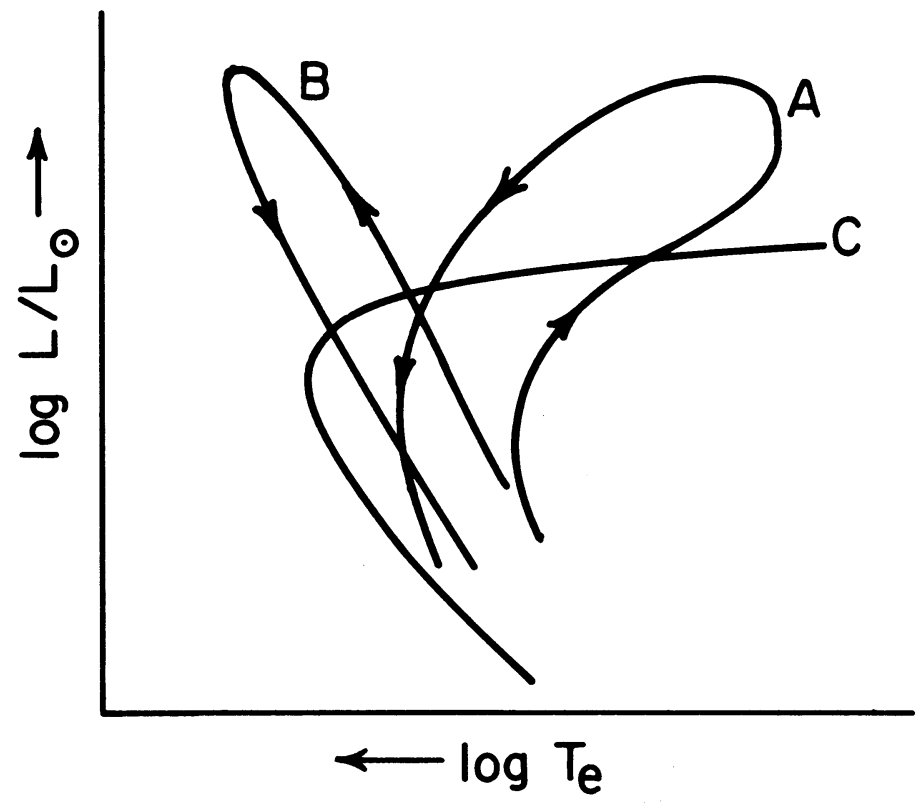

Figure 4. The effect of the initial conditions. Curves A and B correspond to a nondegenerate initial core; A with a large amount of hydrogen, B with a small amount of hydrogen. Curve $\mathrm{C}$ is for models in thermal equilibrium and a very small amount of $\mathrm{H}$.

Finally, we remark on the coincidence between the expansion of the nebula and the heating of the CNP to above $40,000{ }^{\circ} \mathrm{K}$. If the mass of the nebula is large and the star stays a short time at a phase with $\mathrm{T}_{\mathrm{e}}>4 \times 10^{40 \mathrm{~K}}$, there will not be enough $\mathrm{L} \alpha$ photons to ionize the whole nebula. And vice versa, if the mass of the nebula is small and moves fast and the CPN is very luminous, our chances of finding the object are again smal1. Consequently, there might be other cases in which the times do not match so well and escape observation. The nice agreement between the birthrate of PN and death rate of MS stars does not allow more than a factor of about 2 between observed and unobserved nebula.

Clearly, if the stars are making too many loops in the HR diagram, the HS will become a locus and not a sequence. However, if the stars stay a significant amount of time in a loop we might have a mixed situation: the HS is mostly an evolutionary sequence but a certain section of it is a locus of flashing stars.

\section{REFERENCES}

Deinzer, W. and Sengburch, K.V. (1970) Ap.J. 160, 671.

O'Del1, C.R. (1963), Ap.J. 138, 67.

Seaton, M. (1966), M.N.R.A.S. $132,113$.

Harman, R.J. and Seaton, M. (1964), Ap.J. 140, 824 .

Joss, P.C.; Katz, J.I.; Malone, R.C. and Salpeter, E.E. (1973), Ap.J., 
$181,409$.

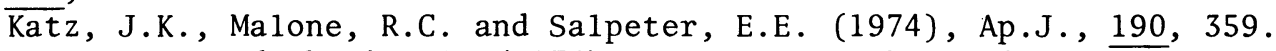

Kovetz, A. and Shaviv, G. (1976) Astron. Astrophys. 52, 403.

Paczyński, B., (1970), Acta Astr. 20, 47, (1972), ib̄id. 27, 417.

Härm, R. and Schwarzschild, M. (1975), Ap.J., 200, $\overline{324}$. 